\begin{tabular}{|c|c|}
\hline \multirow{3}{*}{ 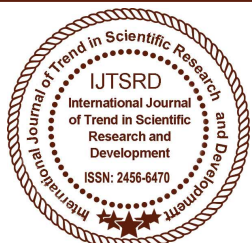 } & International Journal of Trend in Scientific Research and Development (IJTSRD) \\
\hline & International Open Access Journal | www.ijtsrd.com \\
\hline & ISSN No: 2456 - 6470 | Volume - 2 | Issue - 6 | Sep-Oct 2018 \\
\hline
\end{tabular}

\title{
Optical Soliton Simulation by Symmetrized Split-Step Fourier Method
}

\author{
K. G. Tay \\ Lecturer, Communication \\ Department, Faculty of Electrical \\ and Electronic, University Tun \\ Hussein Onn Malaysia, Johor, \\ Malaysia
}

\author{
W. K. Tiong \\ Lecturer, Department of \\ Computational Science and \\ Mathematics, University of \\ Malaysia Sarawak, Sarawak, \\ Malaysia
}

\author{
Y. Y. Choy \\ Lecturer, Department of \\ Mathematics and Statistics, \\ Faculty of Applied Sciences and \\ Technology, University Tun \\ Hussein Onn Malaysia, Johor, \\ Malaysia
}

\begin{abstract}
Fiber optic telecommunication gives more benefits if compared to copper wire communication systems, however, group velocity dispersion (GVD), fiber loss and also self-phase modulation (SPM) limit the performance of fiber optic telecommunication. When there is a balance between GVD and SPM, there exists a stable wave (optical soliton) which can propagate for long distance undistorted. In this study, the optical soliton simulation will be carried out using symmetrized split-step Fourier method (SSFM).
\end{abstract}

Keyword: Optical soliton, symmetrized split-step Fourier method, SSFM, GVD, SPM

\subsection{INTRODUCTION}

Modern people today cannot live without the Internet. They require higher bandwidth and faster internet speed connection for job, educational, entertainment and social purposes. Therefore, a fiber optic is utilized in ultra-fast long-haul telecommunication. Fiber optic telecommunication uses light to transfer information through an optical fiber. The optical fiber is much smaller and lighter than copper wire. It provides a broader bandwidth, higher frequency transmission of signals with a greater bit rate over a long distance with lower loss and lower interference if compared to the copper wire communication systems. But, group velocity dispersion (GVD), fiber loss and also selfphase modulation (SPM) limit the performance of fiber optic telecommunication. [1]
GVD or Chromatic Dispersion in the optical fiber is due to the refractive index of the fiber is frequency dependent and hence light of different frequencies travel along the fiber at different velocities. Thus they arrive at the receiver at different times. This result in pulse spreading which cause intersymbol interference hence signals may not be detected correctly. [2]

The Kerr effect due to fiber nonlinearity which is dependent on refractive index variation due to the intensity of light causes SPM. SPM produces a change of spectrum without change of temporal distribution.

When there is a balance between dispersion broadening due to GVD and SPM in an optical fiber, a special kind of wave called temporal optical soliton (confinement of light occurs in time) is formed. When confinement of light happens in space, a spatial soliton is formed. Soliton can travel steadily for long distance (thousands of $\mathrm{km}$ ) [3] undistorted in a lossless optical fiber. This property makes soliton suitable to be applied in telecommunication.

The optical soliton propagation in fiber optic is governed by the nonlinear Schrodinger (NLS) equation which can be derived by Maxwell equations. [4]

$i \frac{\partial A}{\partial z}-\frac{\beta_{2}}{2} \frac{\partial^{2} A}{\partial t^{2}}+\gamma|A|^{2} A+\frac{i}{2} \alpha A=0$ 


\subsection{Symmetrized Split Step Fourier method (SSFM)}

The NLS Equation (1) will be solved numerically by numerical method namely Symmetrized SSFM.

$\frac{\partial A}{\partial z}=-i \frac{\beta_{2}}{2} \frac{\partial^{2} A}{\partial t^{2}}+i \gamma|A|^{2} A-\frac{1}{2} \alpha A$

Firstly, Equation (2) is divided into linear and nonlinear operator such that

$$
\frac{\partial A}{\partial z}=(\hat{L}+\hat{N}) A
$$

Where $\hat{L}, \hat{N}$ are linear and nonlinear operators.

In this case. $\hat{L}=-\frac{i \beta_{2}}{2} \frac{\partial^{2}}{\partial t^{2}}-\frac{1}{2} \alpha, \quad \hat{N}=i \gamma|A|^{2}$

The solution of Equation (3) is

$$
\begin{aligned}
& \frac{\partial A}{A}=(\hat{L}+\hat{N}) \partial z \\
& \operatorname{Ln}(A)=(\hat{L}+\hat{N}) z+c \\
& A(z+\Delta z, t)=\exp [(\hat{L}+\hat{N}) \Delta z] A(z, t)
\end{aligned}
$$

The Symmetrized SSFM method will be advanced using half step of a linear operator, then one step of the nonlinear operator and lastly half step of a linear operator as below:

$$
A(z+\Delta z, t)=\exp \left(\frac{\Delta z}{2} \hat{L}\right) \exp \left(\int_{z}^{z+\Delta z} \hat{N}(z) \Delta z^{\prime}\right) \exp \left(\frac{\Delta z}{2} \hat{L}\right) A(z, t)
$$

Where $\Delta z$ is the spatial step and the integral part is solved using the trapezoidal rule.

$$
\int_{z}^{z+\Delta z} \hat{N}(z) \Delta z^{\prime} \approx \frac{\Delta z}{2}[\hat{N}(z)+\hat{N}(z+\Delta z)]
$$

Sub (6) in (5) yields

$$
A(z+\Delta z, t)=\exp \left(\frac{\Delta z}{2} \hat{L}\right) \exp \left(\frac{\Delta z}{2} \hat{N}(z)\right) \exp \left(\frac{\Delta z}{2} \hat{N}(z+\Delta z)\right) \exp \left(\frac{\Delta z}{2} \hat{L}\right) A(z, t)
$$

Simplifying it

$$
\begin{aligned}
& A(z+\Delta z, t)=\exp \left(\frac{\Delta z}{2} \hat{L}\right) \exp \left(\frac{\Delta z[\hat{N}(z)+\hat{N}(z+\Delta z)]}{2}\right) \exp \left(\frac{\Delta z}{2} \hat{L}\right) A(z, t) \\
& A(z+\Delta z, t)=\exp \left(\frac{\Delta z}{2} \hat{L}\right) \exp \left(\Delta z \hat{N}\left(z+\frac{\Delta z}{2}\right)\right) \exp \left(\frac{\Delta z}{2} \hat{L}\right) A(z, t)
\end{aligned}
$$

By taking the Fourier transform (FFT) on Equation (8), we get 
$A(z+\Delta z, t)=F^{-1}\left\{\exp \left(-\frac{\Delta z}{2}\left[\frac{i \beta_{2}}{2}(i w)^{2}+\frac{\alpha}{2}\right]\right) F\left\{\exp \left(\Delta z i \gamma\left|A_{\text {half }}\right|^{2}\right) F^{-1}\left\{\exp \left(-\frac{\Delta z}{2}\left[\frac{i \beta_{2}}{2}(i w)^{2}+\frac{\alpha}{2}\right]\right) F\{A(z, t)\}\right\}\right\}\right\}(9)$

where $A_{\text {half }}=F^{-1}\left\{\exp \left(-\frac{\Delta z}{2}\left[\frac{i \beta_{2}}{2}(i w)^{2}+\frac{\alpha}{2}\right]\right) F\{A(z, t)\}\right\}, \omega$ is the Fourier frequency.

\subsection{Methodology}

In this optical fiber communication link, single mode fiber (SMF) is used to propagate the signal with wavelength, $\lambda=1550 \mathrm{~nm}$. The attenuation, $\alpha$ of single mode fiber is $0 \mathrm{~dB} / \mathrm{km}$, the dispersion, $\beta_{2}$ of this single mode fiber is $-20 \mathrm{ps}^{2} / \mathrm{km}$. and the parameter for nonlinearity, $\gamma$ is $1.317 \mathrm{~W}^{-1} \mathrm{~km}^{-1}$. At $40 \mathrm{~GB} / \mathrm{s}$ bit rate, the bit slot is $25 \mathrm{ps}$ as shown below [5]:

Time window $($ bit slot $)=$ Sequence length $\mathrm{x} \frac{1}{\text { Bit rate }}$

$$
\begin{aligned}
& =1 \times \frac{1}{40 G} \\
& =25 \mathrm{ps} .
\end{aligned}
$$

The pulse full width half maximum (FWHM), $T_{F W H M}$ is $12.5 \mathrm{ps}$. The relation between the temporal characteristicvalue of the initial pulse of $T_{0}$ parameter and $T_{F W H M}$ for the sech pulses is

$T_{F W H M}=2 \ln (1+\sqrt{2}) T_{0}$

$\cong 1.763 T_{0}$

Hence initial pulse of $T_{0}$ is

$$
\begin{aligned}
& T_{0}=\frac{T_{F W H M}}{1.763} \\
& =\frac{12.5 \mathrm{ps}}{1.763} \\
& =7.0902 \mathrm{ps} .
\end{aligned}
$$

The formula of power is:

$$
\begin{aligned}
& P=\frac{\left|\beta_{2}\right|}{N^{2} \gamma T_{0}{ }^{2}} \\
& =\frac{(|20|)}{(1.317)(7.0902)^{2}} \\
& =0.30208 \mathrm{~N}^{2}[\mathrm{~W}]
\end{aligned}
$$

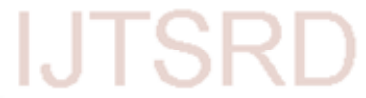

For fundamental soliton, the soliton number $N$ is equal to one.

The dispersion length is given as:

$$
\begin{aligned}
& L_{D}=\frac{T o^{2}}{\left|\beta_{2}\right|} \\
& =\frac{7.0902 \mathrm{ps}^{2}}{20 \mathrm{ps} 2 / \mathrm{km}} \\
& =2.5135 \mathrm{~km}
\end{aligned}
$$

Whereas the nonlinearity length is defined as:

$$
\begin{aligned}
& L_{N L}=\frac{1}{\gamma P_{0}} \\
& =\frac{1}{\left(1.317 \mathrm{~W}^{-1} \mathrm{~km}^{-1} .\right)(0.30208)} \\
& =2.5135 \mathrm{~km}
\end{aligned}
$$

The soliton period is given by

$$
\begin{aligned}
& \mathrm{z}_{0}=\frac{\pi}{2}\left(L_{D}\right) \\
& =\frac{\pi}{2}(2.5135) \\
& =3.9482 \mathrm{~km} .
\end{aligned}
$$




\subsection{Results and Discussions}

The initial wave at $z=0 \mathrm{~km}$ of the optical soliton was inputted into the symmetrized SSFM scheme (9) as given in Figure 1. It is noticed that the amplitude is given by $P$ and the pulse width is given by $\frac{t}{T_{0}}$. The initial pulse is in sech form. The optical soliton propagates along the optical fiber at one soliton period of $\mathrm{z}_{0}=3.9483 \mathrm{~km}$ and it was round up to $4 \mathrm{~km}$.

The 3D-visualization of the optical soliton propagation is depicted in Figure 1.The results of optical soliton propagation at $z=1 \mathrm{~km}, z=2 \mathrm{~km}, z=3 \mathrm{~km}$ and $z=4 \mathrm{~km}$ are shown in Figures $2-5$ respectively. While Figure 7 gives a series of 2D-graph at each distance of $z$ obtained from Simbiolgy in Matlab.It can be seen that the optical soliton propagates undistorted (amplitude and shape maintain) along the optical fiber.

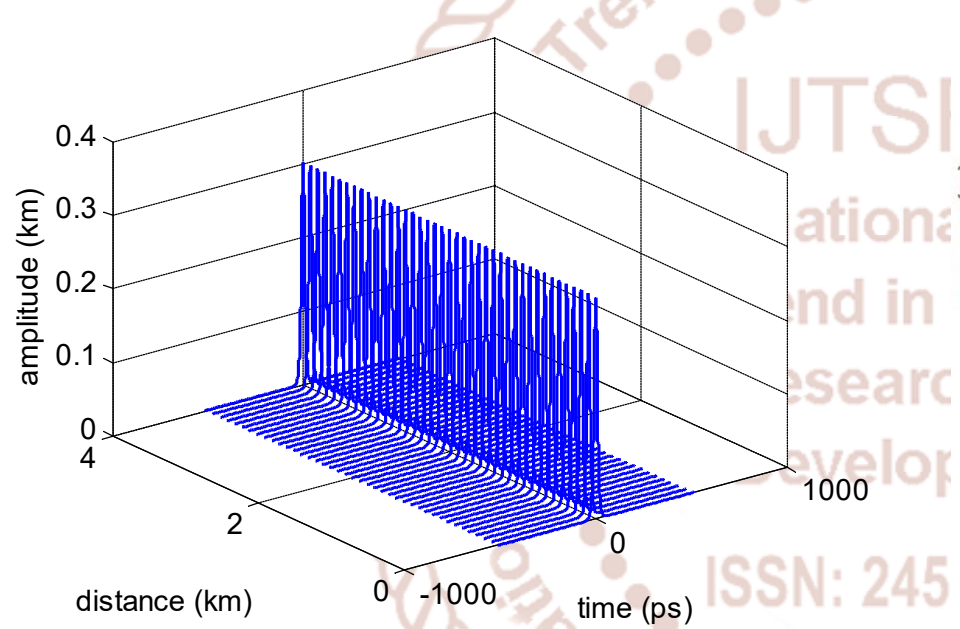

Figure 1: 3D-Optical soliton

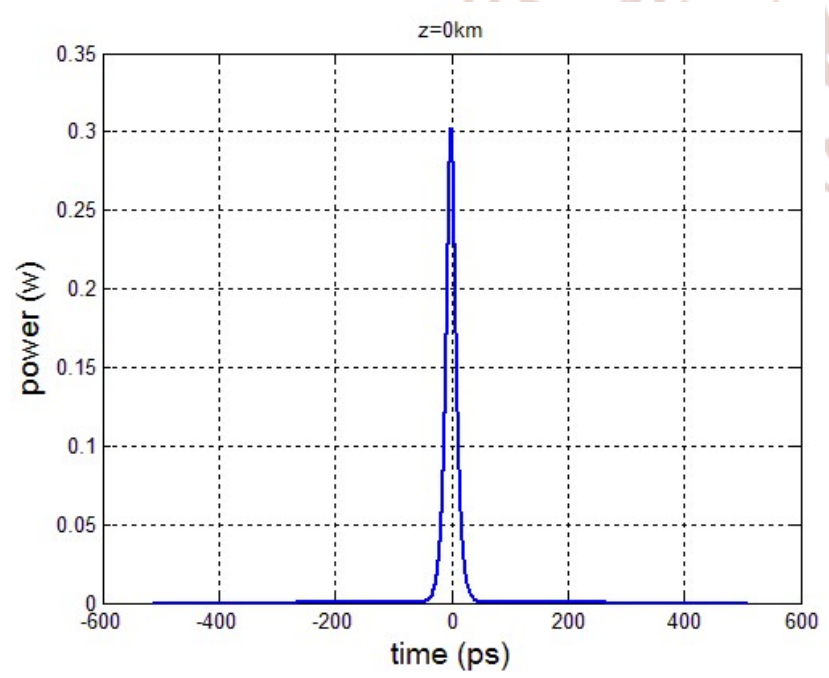

Figure 2: Initial optical soliton at $z=0 \mathrm{~km}$

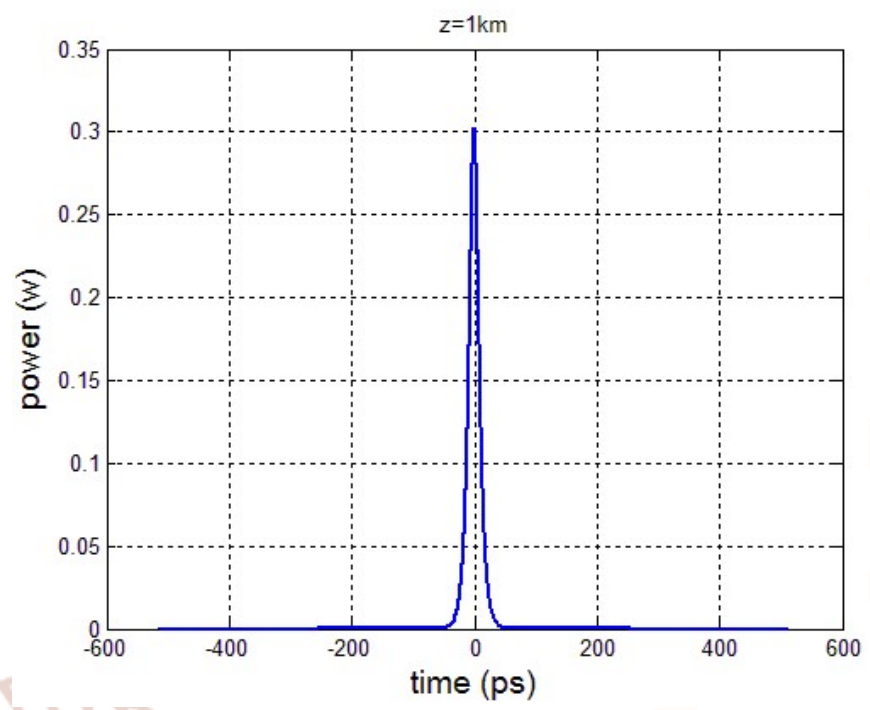

Figure 3: Optical soliton at $z=1 \mathrm{~km}$

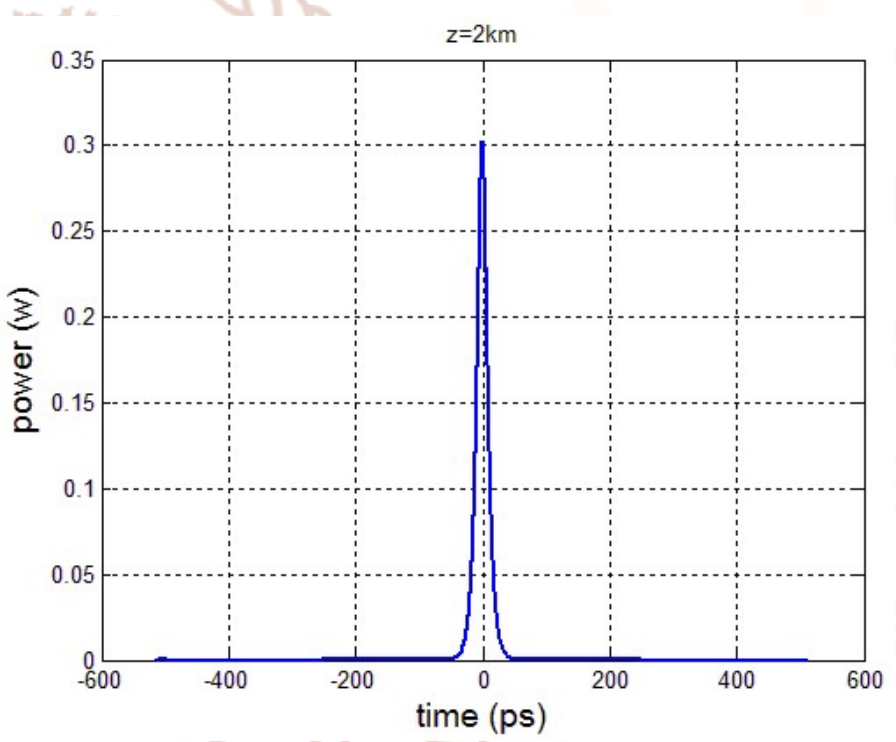

Figure 4: Optical soliton at $z=2 \mathrm{~km}$

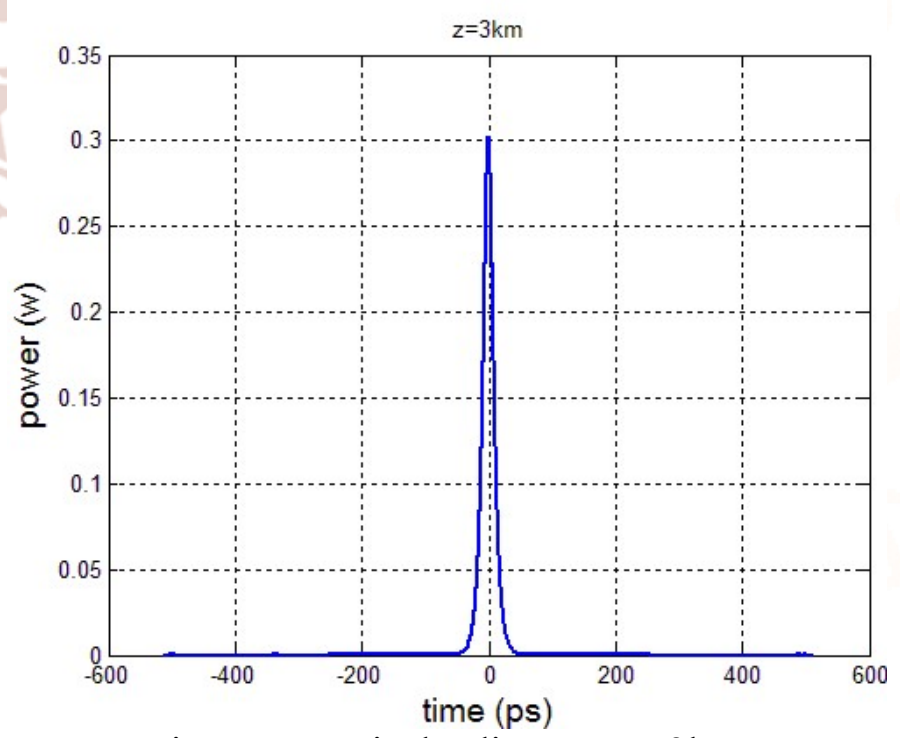

Figure 5: Optical soliton at $z=3 \mathrm{~km}$ 


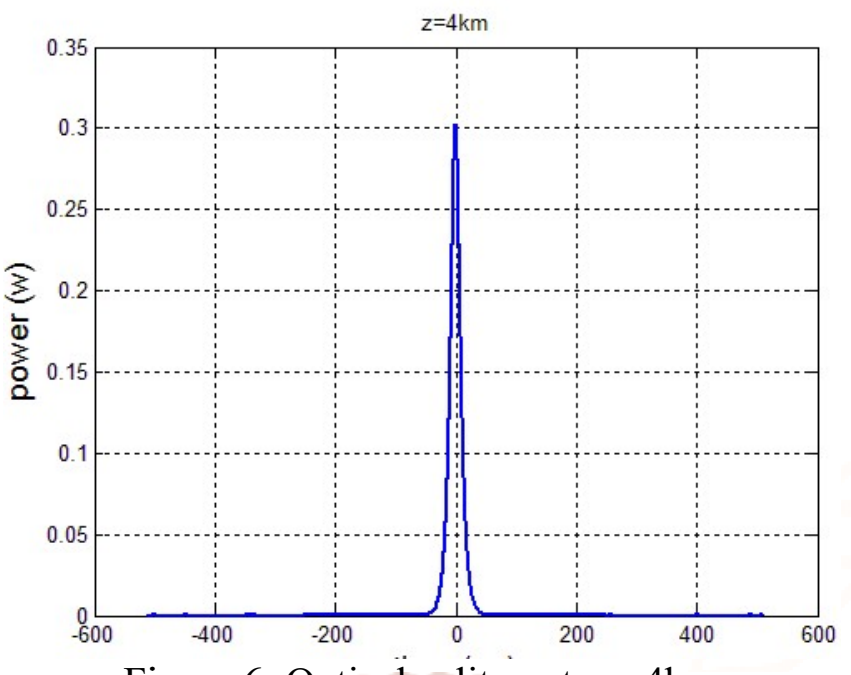

Figure 6: Optical soliton at $z=4 \mathrm{~km}$

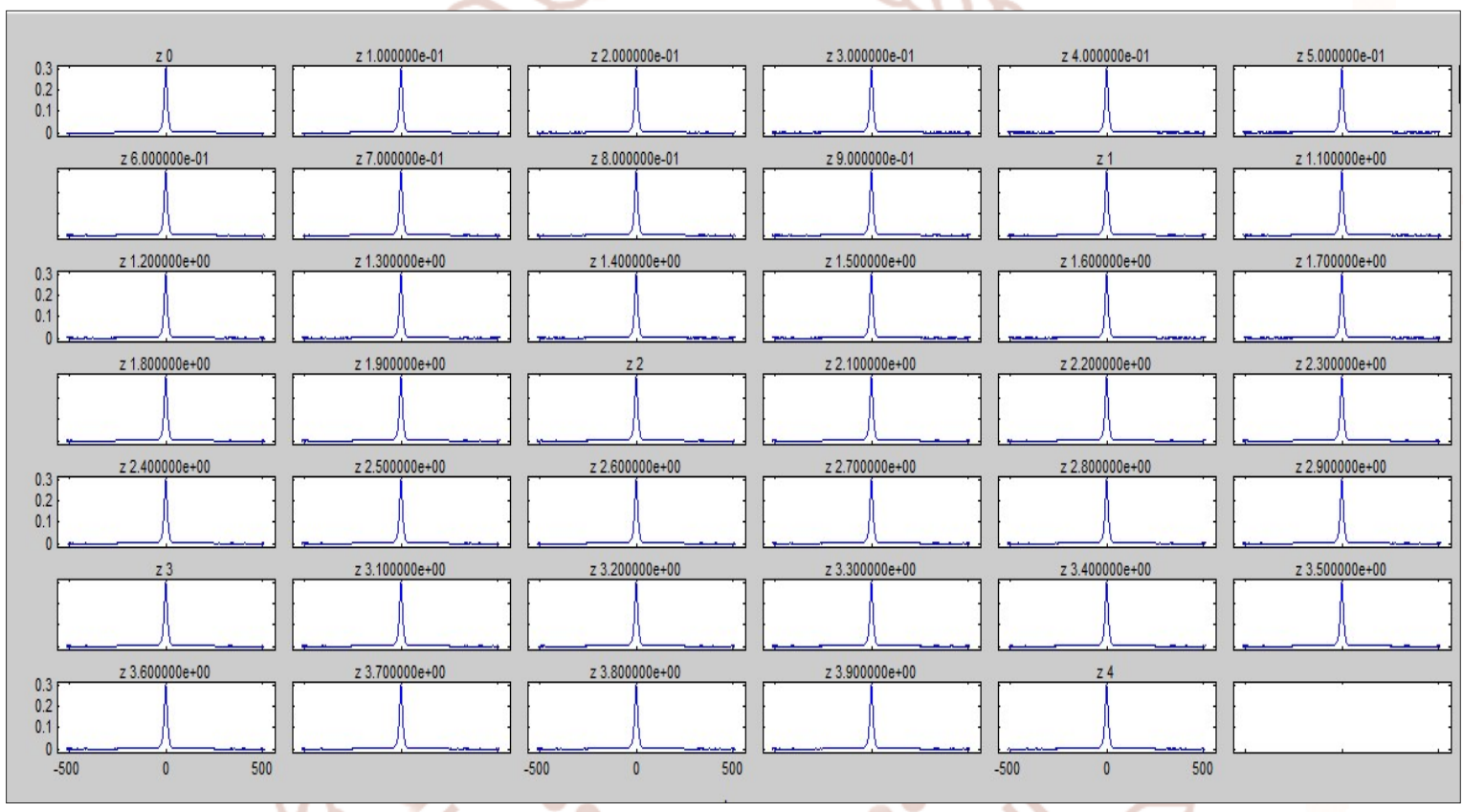

Figure 7: Series of 2D Optical soliton for one soliton period

\subsection{Conclusion}

The optical soliton propagation along the optical fiber with $\beta_{2}=-20 \quad \mathrm{ps}^{2} / \mathrm{km}, \quad \gamma=1.317 \mathrm{~W}^{-1} \mathrm{~km}^{-1}$ and $\alpha=\mathrm{dB} / \mathrm{km}$ was simulated. The optical soliton can propagate for long distance while maintaining its shape and amplitude.

\section{References}

1. Tay, K. G., Audrey, H. K. C., Loi. w. S. Ong C. T. 2017. 'Optical Soliton Simulation in Optical Fibers by OptiSystem". IOP Conf. Series: Materials Science and Engineering, 226:1-12.

2. Nair, S. S. and Prem, V. 2014. "Solitons: A Promising Technology in Optical Communication". International Journal of Science and Research (IJSR), 3:151--154.
3. Sharma, R. L and Singh, R. 2011. 'Solitons, its Evolution and Applications in High Speed Optical Communication". International Journal on Emerging Technologies, 2:141--145.

4. Agrawal, G. P. 2001. Nonlinear Fiber Optics. London: Academic Press.

5. 2008. OptiSystem Tutorials-Volume 2 Optical Communication System Design Software. Canada: Opti Wave. 